\title{
Generalized Abel Inversion Using Extended Hat Functions Operational Matrix
}

\author{
Manoj P. Tripathi, ${ }^{1,2}$ Ram K. Pandey, ${ }^{1}$ Vipul K. Baranwal, ${ }^{1}$ and Om P. Singh ${ }^{1}$ \\ ${ }^{1}$ Department of Applied Mathematics, Indian Institute of Technology, Banaras Hindu University, Varanasi 221005, India \\ ${ }^{2}$ Department of Mathematics, Udai Pratap Autonomous College, Varanasi 221002, India \\ Correspondence should be addressed to Om P. Singh; singhom@gmail.com
}

Received 24 September 2012; Accepted 2 April 2013

Academic Editor: Frédéric Robert

Copyright (C) 2013 Manoj P. Tripathi et al. This is an open access article distributed under the Creative Commons Attribution License, which permits unrestricted use, distribution, and reproduction in any medium, provided the original work is properly cited.

Abel type integral equations play a vital role in the study of compressible flows around axially symmetric bodies. The relationship between emissivity and the measured intensity, as measured from the outside cylindrically symmetric, optically thin extended radiation source, is given by this equation as well. The aim of the present paper is to propose a stable algorithm for the numerical inversion of the following generalized Abel integral equation: $I(y)=a(y) \int_{\alpha}^{y}\left(\left(r^{\mu-1} \varepsilon(r)\right) /\left(y^{\mu}-r^{\mu}\right)^{\gamma}\right) d r+b(y) \int_{y}^{\beta}\left(\left(r^{\mu-1} \varepsilon(r)\right) /\left(r^{\mu}-\right.\right.$ $\left.\left.y^{\mu}\right)^{\gamma}\right) d r, \alpha \leq y \leq \beta, 0<\gamma<1$, using our newly constructed extended hat functions operational matrix of integration, and give an error analysis of the algorithm. The earlier numerical inversions available for the above equation assumed either $a(y)=0$ or $b(y)=0$.

\section{Introduction}

Abel integral equation [1] occurs in many branches of science and technology, such as plasma diagnostics and flame studies, where the most common problem of deduction of radial distributions of some important physical quantity from measurement of line-of-sight projected values is encountered. For a cylindrically symmetric, optically thin plasma source, the relation between radial distribution of the emission coefficient and the intensity measured from outside of the radial source is described by Abel transform. The challenging task of reconstruction of emission coefficient from its projection is known as Abel inversion. The earliest application, due to Mach [2], arose in the study of compressible flows around axially symmetric bodies.

The Abel integral equation is given by

$$
I(y)=2 \int_{y}^{1} \frac{\varepsilon(r) r}{\sqrt{r^{2}-y^{2}}} d r, \quad 0 \leq y \leq 1,
$$

where $\varepsilon(r)$ and $I(y)$ represent, respectively, the emissivity and measured intensity, as measured from outside the source [3].
The analytical inversion formula for (1) is given as [4]

$$
\varepsilon(r)=-\frac{1}{\pi} \int_{r}^{1} \frac{1}{\sqrt{y^{2}-r^{2}}} \frac{d I(y)}{d(y)} d y, \quad 0 \leq r \leq 1 .
$$

There are several analytic and numerical inversion formulae available in the literature $[1,5-20]$.

Singh et al. [19] constructed an operational matrix of integration based on orthonormal Bernstein polynomials and used it to propose a stable algorithm to invert the following form of Abel integral equation:

$$
I(y)=2 \int_{0}^{y} \frac{\varepsilon(r) r}{\sqrt{r^{2}-y^{2}}} d r, \quad 0 \leq y \leq 1 .
$$

In 2010, Singh et al. [20] constructed yet another operational matrix of integration based on orthonormal Bernstein polynomials and used it to propose an algorithm to invert the Abel integral equation (1). 
In 2008, Chakrabarti [21] employed a direct function theoretic method to determine the closed form solution of the following generalized Abel integral equation:

$$
\begin{array}{r}
I(y)=a(y) \int_{\alpha}^{y} \frac{r^{\mu-1} \varepsilon(r)}{\left(y^{\mu}-r^{\mu}\right)^{\gamma}} d r+b(y) \int_{y}^{\beta} \frac{r^{\mu-1} \varepsilon(r)}{\left(r^{\mu}-y^{\mu}\right)^{\gamma}} d r, \\
\alpha \leq y \leq \beta, \quad 0<\gamma<1,
\end{array}
$$

where the coefficients $a(y)$ and $b(y)$ do not vanish simultaneously. But the numerical inversion is still needed for its application in physical models since the experimental data for the intensity $I(y)$ is available only at a discrete set of points, and it may also be distorted by the noise.

This motivated us to look for a stable algorithm which can be used for numerical inversion of the Abel integral equation (4) obtained by joining the two integrals (1) and (3). In this paper, we construct extended hat functions operational matrix of integration to invert the generalized Abel integral equation (4). Using hat functions for approximation of emissivity and intensity profiles has an edge over the earlier works of Singh et al. [19, 20], where they have used orthonormal Bernstein polynomials to approximate those physical quantities in the sense that a general formula for $n \times n$ operational matrix of integration is obtained in the earlier case whereas no such formula is available for the latter case. In Sections 3 and 4, we give the error estimate and the stability analysis followed by numerical examples to illustrate the efficiency and stability of the proposed algorithm.

The above two forms (1) and (3) of Abel integral equations are obtained by taking $\gamma=1 / 2$ and

(i) $a(y)=0, b(y)=2, \beta=1, \mu=2$;

(ii) $a(y)=2, b(y)=0, \alpha=0, \mu=1$, respectively, in (4).

Mostly for $\mu=1,2$ and $\gamma=1 / 2$ the generalized Abel integral equation models the physical problems but the integral equation for $\mu=2$ can be reduced to the case $\mu=1$, by change of variables. So we restrict ourselves to $\mu=1$ only.

\section{Extended Hat Functions and Their Operational Matrices for Abel Inversion}

Hat functions are defined on the domain $[0,1]$. These are continuous functions with shape of hats, when plotted on two-dimensional planes. The interval $[0,1]$ is divided into $n$ subintervals $[i h,(i+1) h], i=0,1,2, \ldots, n-1$, of equal lengths $h$ where $h=1 / n$. The hat function's family of first $(n+1)$ hat functions is defined as follows:

$$
\psi_{0}(t)= \begin{cases}\frac{h-t}{h}, & 0 \leq t<h \\ 0, & \text { otherwise }\end{cases}
$$

$$
\begin{gathered}
\psi_{i}(t)= \begin{cases}\frac{t-(i-1) h}{h}, & (i-1) h \leq t<i h, \\
\frac{(i+1) h-t}{h}, & i h \leq t<(i+1) h, \\
0, & i=1,2, \ldots, n-1,\end{cases} \\
\psi_{n}(t)= \begin{cases}\frac{t-(1-h)}{h}, & 1-h \leq t \leq 1, \\
0, & \text { otherwise, }\end{cases}
\end{gathered}
$$

We modify these functions by adding characteristics functions $\chi_{[-\tau, 0)}$ and $\chi_{(1,1+\tau]}$ to $\psi_{0}$ and $\psi_{n}$, respectively, to yield a new class of extended hat functions $\bar{\psi}_{i}(t)$ defined over $[-\tau, 1+$ $\tau$ ] for $\tau>0$, and these are given by

$$
\begin{gathered}
\bar{\psi}_{0}(t)=\chi_{[-\tau, 0)}(t)+\psi_{0}(t), \\
\bar{\psi}_{i}(t)=\psi_{i}(t), \quad \text { for } 1 \leq i \leq n-1, \\
\bar{\psi}_{n}(t)=\psi_{n}(t)+\chi_{(1,1+\tau]}(t) .
\end{gathered}
$$

Thus, the supports of $\bar{\psi}_{0}(t)$ and $\bar{\psi}_{n}(t)$ are extended to $[-\tau, h]$ and $[1-h, 1+\tau]$, respectively. These extended hat functions $\bar{\psi}_{j}(t)$ are continuous, linearly independent and are in $L^{2}[-\tau, 1+\tau]$. As $\tau \rightarrow 0$, obviously, the extended hat functions will converge to the traditional hat functions.

A function $f \in L^{2}[0,1]$ may be approximated in vector form as

$$
f(t) \simeq \sum_{i=0}^{i=n} f_{i} \bar{\psi}_{i}(t)=F_{n+1}^{T} \bar{\Psi}_{n+1}(t)=\bar{\Psi}_{n+1}^{T}(t) F_{n+1},
$$

where

$$
\begin{gathered}
F_{n+1} \triangleq\left[f_{0}, f_{1}, f_{2}, \ldots, f_{n}\right]^{T}, \\
\bar{\Psi}_{n+1}(t) \triangleq\left[\bar{\psi}_{0}(t), \bar{\psi}_{1}(t), \bar{\psi}_{2}(t), \ldots, \bar{\psi}_{n}(t)\right]^{T} .
\end{gathered}
$$

The important aspect of using extended hat functions in the approximation of function $f(t)$ lies in the fact that the coefficients $f_{i}$ in (11) are given by

$$
f_{i}=f(i h), \quad i=0,1,2 \ldots, n .
$$

Taking $\alpha=0, \beta=1$, and $\mu=2$ and by change of variables, the Abel integral equation (4) reduces to

$$
\begin{array}{r}
I(\sqrt{y})=\frac{a(\sqrt{y})}{2} \int_{0}^{y} \frac{\varepsilon(\sqrt{r})}{(y-r)^{\gamma}} d r+\frac{b(\sqrt{y})}{2} \int_{y}^{1} \frac{\varepsilon(\sqrt{r})}{(r-y)^{\gamma}} d r, \\
0 \leq y \leq 1,
\end{array}
$$

which may be written as

$$
\begin{array}{r}
I_{1}(y)=a_{1}(y) \int_{0}^{y} \frac{\eta(r)}{(y-r)^{\gamma}} d r+b_{1}(y) \int_{y}^{1} \frac{\eta(r)}{(r-y)^{\gamma}} d r, \\
0 \leq y \leq 1,
\end{array}
$$


where $I_{1}(y)=I(\sqrt{y}), \eta(r)=\varepsilon(\sqrt{r}), a_{1}(y)=a(\sqrt{y}) / 2$, and $b_{1}(y)=b(\sqrt{y}) / 2$.

Instead of considering (15), we consider the more general equation of the form:

$$
\begin{array}{r}
I_{1}(y)=a_{1}(y) \int_{-\tau}^{y} \frac{\eta(r)}{(y-r)^{\gamma}} d r+b_{1}(y) \int_{y}^{1+\tau} \frac{\eta(r)}{(r-y)^{\gamma}} d r \\
0 \leq y \leq 1 .
\end{array}
$$

Using (11), the functions $I_{1}(y)$ and $\eta(r)$ may be approximated as

$$
I_{1}(y) \simeq F_{n+1}^{T} \bar{\Psi}_{n+1}(y), \quad \eta(r) \simeq C_{n+1}^{T} \bar{\Psi}_{n+1}(r)
$$

Thus the problem of Abel inversion is reduced to finding the unknown matrix $C_{n+1}$. Substituting (17) into (16), we get

$$
\begin{array}{r}
F_{n+1}^{T} \bar{\Psi}_{n+1}(y) \\
=C_{n+1}^{T}\left[a_{1}(y) \int_{-\tau}^{y} \frac{\bar{\Psi}_{n+1}(r)}{(y-r)^{\gamma}} d r+b_{1}(y) \int_{y}^{1+\tau} \frac{\bar{\Psi}_{n+1}(r)}{(r-y)^{\gamma}} d r\right], \\
0 \leq y \leq 1 .
\end{array}
$$

The integrals in (18) involve, evaluating integrals of the type $\int_{y}^{1+\tau}\left(\left(\bar{\Psi}_{n+1}(r)\right) /(r-y)^{\gamma}\right) d r$ and $\int_{-\tau}^{y}\left(\left(\bar{\psi}_{i}(r)\right) /(y-r)^{\gamma}\right) d r$. Let

$$
\begin{array}{r}
\Phi_{i}^{L}(y)=\int_{y}^{1+\tau} \frac{\bar{\psi}_{i}(r)}{(r-y)^{\gamma}} d r, \quad \Phi_{i}^{U}(y)=\int_{-\tau}^{y} \frac{\bar{\psi}_{i}(r)}{(y-r)^{\gamma}} d r \\
\text { for } i=0,1,2 \ldots, n,
\end{array}
$$

and compute the two operational matrices of integration to evaluate these integrals. The scheme of derivation of these two operational matrices is based on the following theorems.

Theorem 1. The functions $\Phi_{i}^{L}(y) \in L^{2}[-\tau, 1+\tau]$ for $i=$ $0,1,2, \ldots, n$.

Proof. We prove the theorem for $i=1,2 \ldots, n-1$. The proofs for $i=0$ and $i=n$ are skipped as they may be proved on the same pattern. Based on subdivision of interval $[-\tau, 1+\tau]$, we calculate $\Phi_{i}^{L}(y)$ by considering the following cases. (i) When $-\tau \leq y<(i-1) h$, then

$$
\begin{aligned}
\Phi_{i}^{L}(y)= & \int_{y}^{1+\tau} \frac{\bar{\psi}_{i}(r)}{(r-y)^{\gamma}} d r \\
= & \int_{y}^{(i-1) h} \frac{\bar{\psi}_{i}(r)}{(r-y)^{\gamma}} d r+\int_{(i-1) h}^{i h} \frac{\bar{\psi}_{i}(r)}{(r-y)^{\gamma}} d r \\
& +\int_{i h}^{(i+1) h} \frac{\bar{\psi}_{i}(r)}{(r-y)^{\gamma}} d r+\int_{(i+1) h}^{1+\tau} \frac{\bar{\psi}_{i}(r)}{(r-y)^{\gamma}} d r \\
= & \int_{(i-1) h}^{i h} \frac{\bar{\psi}_{i}(r)}{(r-y)^{\gamma}} d r+\int_{i h}^{(i+1) h} \frac{\bar{\psi}_{i}(r)}{(r-y)^{\gamma}} d r
\end{aligned}
$$

(as the support of $\bar{\psi}_{i}(t)$ lies in $[(i-1) h,(i+1) h]$ )

$$
=\int_{(i-1) h}^{i h} \frac{r-(i-1) h}{h(r-y)^{\gamma}} d r+\int_{i h}^{(i+1) h} \frac{(i+1) h-r}{(r-y)^{\gamma}} d r .
$$

Changing the variable, $r-y=t$, we get

$$
\begin{gathered}
\Phi_{i}^{L}(y)=\frac{1}{h(1-\gamma)(2-\gamma)}\left[((i-1) h-y)^{2-\gamma}-2(i h-y)^{2-\gamma}\right. \\
\left.+((i+1) h-y)^{2-\gamma}\right] .
\end{gathered}
$$

(ii) When $(i-1) h \leq y<i h$, then

$$
\Phi_{i}^{L}(y)=\int_{y}^{i h} \frac{\bar{\psi}_{i}(r)}{(r-y)^{\gamma}} d r+\int_{i h}^{(i+1) h} \frac{\bar{\psi}_{i}(r)}{(r-y)^{\gamma}} d r .
$$

Adopting the same procedure as in (i), we get

$$
\begin{aligned}
\Phi_{i}^{L}(y)= & \frac{1}{h(1-\gamma)(2-\gamma)} \\
& \times\left[((i+1) h-y)^{2-\gamma}-2(i h-y)^{2-\gamma}\right] .
\end{aligned}
$$

(iii) When $i h \leq y<(i+1) h$, then

$$
\begin{aligned}
\Phi_{i}^{L}(y) & =\int_{y}^{(i+1) h} \frac{\bar{\psi}_{i}(r)}{(r-y)^{\gamma}} d r=\int_{y}^{(i+1) h} \frac{(i+1) h-r}{h(r-y)^{\gamma}} d r \\
& =\frac{1}{h(1-\gamma)(2-\gamma)}\left[((i+1) h-y)^{2-\gamma}\right] .
\end{aligned}
$$

(iv) When $(i+1) h \leq y \leq 1+\tau$, then $\Phi_{i}^{L}(y)=0$. Hence

$$
\Phi_{i}^{L}(y)=\frac{1}{h(1-\gamma)(2-\gamma)} \begin{cases}((i-1) h-y)^{2-\gamma}-2(i h-y)^{2-\gamma}+((i+1) h-y)^{2-\gamma}, & -\tau \leq y<(i-1) h, \\ ((i+1) h-y)^{2-\gamma}-2(i h-y)^{2-\gamma}, & (i-1) h \leq y<i h, \\ ((i+1) h-y)^{2-\gamma}, & \text { ih } y<(i+1) h, \\ 0, & \text { otherwise. }\end{cases}
$$


Thus, from (25), we see that $\left\|\Phi_{i}^{L}(y)\right\|_{2}<\infty$ and hence $\Phi_{i}^{L}(y) \in L^{2}[-\tau, 1+\tau]$ for $\tau>0$ and bounded. This completes the proof.

Therefore, from (11), we get

$$
\Phi_{i}^{L}(y) \simeq \sum_{j=0}^{n} c_{i j} \bar{\psi}_{j}(y), \quad \text { for } i=0,1,2 \ldots, n .
$$

Theorem 2. The coefficients $c_{i j}$ in (26) are given by

(i) for $i=0,1,2, \ldots, n-1, j=0,1,2 \ldots, n$,

$$
c_{i j}= \begin{cases}\beta, & \text { for } j=i, \\ \beta\left[(i-j+1)^{2-\gamma}-2(i-j)^{2-\gamma}\right. & \\ \left.0,+(i-j-1)^{2-\gamma}\right], & \text { for } j<i, \\ 0, & \text { for } j>i,\end{cases}
$$

(ii) for $i=n, j=0,1,2, \ldots, n$,

$c_{n j}$

$$
= \begin{cases}\beta\left[(n-j-1)^{2-\gamma}-(n-j)^{2-\gamma}+(2-\gamma)(n-j)^{1-\gamma}\right] & \\ +\frac{1}{(1-\gamma)}\left[(1+\tau-j h)^{1-\gamma}-(1-j h)^{1-\gamma}\right], & \text { for } j<n, \\ \frac{1}{(1-\gamma)} \tau^{1-\gamma}, & \text { for } j=n,\end{cases}
$$

where $\beta=h^{1-\gamma} /((1-\gamma)(2-\gamma))$.

Proof. (i) When $j=i, c_{i i}=\Phi_{i}^{L}(i h)=\beta$ follows from (25).

When $j<i$, which is equivalent to $j \leq i-1$, we get

$$
\begin{aligned}
c_{i j} & =\Phi_{i}^{L}(j h)=\int_{j h}^{1+\tau} \frac{\bar{\psi}_{i}(r)}{(r-j h)^{\gamma}} d r \\
& =\left(\int_{(i-1) h}^{i h}+\int_{i h}^{(i+1) h}\right) \frac{\bar{\psi}_{i}(r)}{(r-j h)^{\gamma}} d r,
\end{aligned}
$$

since the support of $\bar{\psi}_{i}(t)$ lies in $[(i-1) h,(i+1) h]$. Using (6) and (9), we get from change of variable

$$
c_{i j}=\beta\left[(i-j+1)^{2-\gamma}-2(i-j)^{2-\gamma}+(i-j-1)^{2-\gamma}\right] .
$$

Similarly, for $j>i$, that is, $j \geq i+1, c_{i j}=0$ (it follows trivially from (19)).

(ii) For $j<n$,

$$
c_{n j}=\Phi_{n}^{L}(j h)=\left(\int_{j h}^{1}+\int_{1}^{1+\tau}\right) \frac{\bar{\psi}_{n}(r)}{(r-j h)^{\gamma}} d r .
$$

From (10), we have

$$
c_{n j}=\int_{(n-1) h}^{n h} \frac{(r-(n-1) h)}{h(r-j h)^{\gamma}} d r+\int_{1}^{1+\tau} \frac{1}{(r-j h)^{\gamma}} d r,
$$

so

$$
\begin{aligned}
c_{n j}= & \beta\left[(n-j-1)^{2-\gamma}-2(n-j)^{2-\gamma}+(2-\gamma)(i-j)^{2-\gamma}\right] \\
& +\frac{1}{(1-\gamma)}\left[(1+\tau-j h)^{1-\gamma}-(1-j h)^{1-\gamma}\right] .
\end{aligned}
$$

Similarly, $c_{n n}=\Phi_{n}^{L}(1)=\int_{1}^{1+\tau}\left(\bar{\psi}_{n}(r) /(r-1)^{\gamma}\right)=(1 /(1-$ $\gamma)) \tau^{1-\gamma}$, thus, proving the theorem.

Similar arguments prove the following theorem.

Theorem 3. The functions $\Phi_{i}^{U}(y) \in L^{2}[-\tau, 1+\tau]$ for $i=$ $0,1,2, \ldots, n$.

From Theorem 3 and (11), we have

$$
\Phi_{i}^{U}(y) \simeq \sum_{j=0}^{n} d_{i j} \bar{\psi}_{j}(y)
$$

The coefficients $d_{i j}$ 's are given as follows.

(i) For $i=0,1,2, \ldots, n-1 ; j=0,1,2, \ldots, n$,

$$
d_{i j}= \begin{cases}\beta, & \text { for } j=i, \\ \beta\left[(j-i+1)^{2-\gamma}-2(j-i)^{2-\gamma}\right. & \\ \left.\quad+(j-i-1)^{2-\gamma}\right], & \text { for } j>i, \\ 0, & \text { for } j<i,\end{cases}
$$

and

(ii) for $i=n ; j=0,1,2 \ldots, n$,

$$
d_{n j}= \begin{cases}\frac{1}{(1-\gamma)} \tau^{1-\gamma}, & \text { for } j=0, \\ \beta\left[(j-1)^{2-\gamma}-j^{2-\gamma}+(2-\gamma) j^{1-\gamma}\right] & \\ +\frac{1}{(1-\gamma)}\left[(\tau+j h)^{1-\gamma}-(j h)^{1-\gamma}\right], & \text { for } 1 \leq j \leq n .\end{cases}
$$
as

Using (12) and (26), the following integral may be written

$$
\int_{y}^{1+\tau} \frac{\bar{\Psi}_{n+1}(r)}{(r-y)^{\gamma}} d r=\left[\Phi_{0}^{L}(y), \Phi_{1}^{L}(y), \ldots, \Phi_{n}^{L}(y)\right]^{T} .
$$


Substituting the approximation of $\Phi_{i}^{L}(y)$ from (26) in (37), we get

$$
\int_{y}^{1+\tau} \frac{\bar{\Psi}_{n+1}(r)}{(r-y)^{\gamma}} d r=P_{n+1}^{L} \bar{\Psi}_{n+1}(y),
$$

where $P_{n+1}^{L}$ is a $(n+1) \times(n+1)$ matrix whose $(i+1, j+1)$ th entry is $c_{i j}$, given by (27) and (28) for $i, j=0,1,2, \ldots, n$. The matrix $P_{n+1}^{L}$ is given as

$$
\begin{aligned}
P_{n+1}^{L}= & \frac{h^{1-\gamma}}{(1-\gamma)(2-\gamma)} \\
\times & {\left[\begin{array}{ccccccccc}
1 & 0 & 0 & 0 & 0 & 0 & 0 & 0 \\
\xi_{1} & 1 & 0 & 0 & 0 & 0 & 0 & 0 \\
\xi_{2} & \xi_{1} & 1 & 0 & 0 & 0 & 0 & 0 \\
\xi_{3} & \xi_{2} & \xi_{1} & 1 & 0 & 0 & 0 & 0 \\
\xi_{4} & \xi_{3} & \xi_{2} & \xi_{1} & 1 & 0 & 0 & 0 \\
\cdots & \cdots & \cdots & \cdots & \cdots & 1 & 0 & 0 \\
\xi_{n-1} & \xi_{n-2} & \cdots & \xi_{3} & \xi_{2} & \xi_{1} & 1 & 0 \\
\zeta_{n} & \zeta_{n-1} & \cdots & \zeta_{4} & \zeta_{3} & \zeta_{2} & \zeta_{1} & \zeta_{0}
\end{array}\right]_{(n+1) \times(n+1)} }
\end{aligned}
$$

where

$$
\begin{gathered}
\zeta_{0}=\frac{(2-\gamma)}{h^{1-\gamma}} \tau^{1-\gamma}, \\
\zeta_{s}=\left[(s-1)^{2-\gamma}-s^{2-\gamma}+(2-\gamma) s^{1-\gamma}\right] \\
+\frac{(2-\gamma)}{h^{1-\gamma}}\left[(\tau+s h)^{1-\gamma}-(s h)^{1-\gamma}\right], \quad s=1,2,3, \ldots, n,
\end{gathered}
$$

$\xi_{k}=(k+1)^{2-\gamma}-2 k^{2-\gamma}+(k-1)^{2-\gamma}, \quad k=1,2,3, \ldots, n-1$.

The matrix $P_{n+1}^{L}$ is called extended hat functions lower operational matrix for Abel's inversion.

Remark 4. It is evident from (40) that when $\tau=0$, then $\zeta_{0}=$ 0 , and so the lower triangular matrix $P_{n+1}^{L}$ becomes a singular matrix. In this case, the singularity of the matrix $P_{n+1}^{L}$ makes it redundant for numerical computation since the invertibility of the matrix is required to obtain the solution. To make the matrix $P_{n+1}^{L}$ invertible, we introduced a positive parameter $\tau$ and extend the traditional hat function to the interval $[-\tau, 1+$ $\tau]$.

Similarly, using (19) and (34)-(36), we construct the extended hat functions upper operational matrix for Abel's inversion, $P_{n+1}^{U}$, such that

$$
\int_{-\tau}^{y} \frac{\bar{\Psi}_{n+1}(r)}{(y-r)^{\gamma}} d r=P_{n+1}^{U} \bar{\Psi}_{n+1}(y),
$$

where

$$
\begin{aligned}
P_{n+1}^{U}= & \frac{h^{1-\gamma}}{(1-\gamma)(2-\gamma)} \\
& \times\left[\begin{array}{cccccccc}
\zeta_{0} & \zeta_{1} & \zeta_{2} & \zeta_{3} & \zeta_{4} & \cdots & \zeta_{n-1} & \zeta_{n} \\
0 & 1 & \xi_{1} & \xi_{2} & \xi_{3} & \cdots & \xi_{n-2} & \xi_{n-1} \\
0 & 0 & 1 & \xi_{1} & \xi_{2} & \xi_{3} & \cdots & \xi_{n-2} \\
0 & 0 & 0 & 1 & \xi_{1} & \xi_{2} & \cdots & \xi_{n-3} \\
0 & 0 & 0 & 0 & 1 & \xi_{1} & \cdots & \xi_{n-4} \\
\cdots & \cdots & \cdots & \cdots & \cdots & \cdots & \cdots & \cdots \\
0 & \cdots & \cdots & \cdots & \cdots & 0 & 1 & \xi_{1} \\
0 & \cdots & \cdots & \cdots & \cdots & \cdots & 0 & 1
\end{array}\right]_{(n+1) \times(n+1)}
\end{aligned}
$$

The various entries $\zeta_{0}, \zeta_{s}$, and $\xi_{k}$ are given by (40)-(42), for $k=1,2,3, \ldots, n-1$ and $s=1,2,3, \ldots, n$.

If we partition the matrix $P_{n+1}^{L}$ in four blocks as $P_{n+1}^{L}=\left[\begin{array}{ll}A & O \\ B & \zeta_{0}\end{array}\right]$, then $P_{n+1}^{U}=\left[\begin{array}{ll}\zeta_{0} & B^{\prime} \\ O & A^{T}\end{array}\right]$, where $B=$ $\left[\begin{array}{llll}\zeta_{n} & \cdots & \zeta_{2} & \zeta_{1}\end{array}\right]_{1 \times n}, B^{\prime}=\left[\begin{array}{llll}\zeta_{1} & \zeta_{2} & \cdots & \zeta_{n}\end{array}\right]_{1 \times n}, O$ is a $n \times 1$ null vector, and

$$
A=\left[\begin{array}{cccccc}
1 & 0 & 0 & 0 & 0 & 0 \\
\xi_{1} & 1 & 0 & 0 & 0 & 0 \\
\xi_{2} & \xi_{1} & 1 & 0 & 0 & 0 \\
\xi_{3} & \xi_{2} & \xi_{1} & 1 & 0 & 0 \\
\xi_{n-2} & \cdots & \cdots & \xi_{1} & 1 & 0 \\
\xi_{n-1} & \xi_{n-2} & \cdots & \cdots & \xi_{1} & 1
\end{array}\right]_{n \times n}
$$

Using (38) and (43), (18) may be written as

$$
F_{n+1}^{T} \bar{\Psi}_{n+1}(y)=C_{n+1}^{T}\left[a(y) P_{n+1}^{U}+b(y) P_{n+1}^{L}\right] \bar{\Psi}_{n+1}(y) .
$$

Solving the above system of linear equations, we obtain

$$
C_{n+1}^{T}=F_{n+1}^{T}\left[a(y) P_{n+1}^{U}+b(y) P_{n+1}^{L}\right]^{-1} .
$$

Substituting the value of $C_{n+1}^{T}$ from (47) into (17), the approximate emissivity $\eta(r)$ is given by

$$
\eta(r)=F_{n+1}^{T}\left[a(y) P_{n+1}^{U}+b(y) P_{n+1}^{L}\right]^{-1} \bar{\Psi}_{n+1}(r) .
$$

\section{Error Analysis}

In this section, an error analysis of our proposed algorithm is given. Let $f \in L^{2}[0,1]$, and then, using (11), it is approximated as

$$
f(t) \simeq \sum_{i=0}^{i=n} f_{i} \bar{\psi}_{i}(t)=\sum_{i=0}^{i=n} f(i h) \psi_{i}(t) .
$$


The above approximation gives exact values at nodal points. We denote the right-hand side of (49) by $\widetilde{f}(t)$, and then, for $j h \leq t<(j+1) h, j=0,1, \ldots, n-1$, we have

$$
\begin{aligned}
\tilde{f}(t)= & \sum_{i=0}^{i=n} f(i h) \psi_{i}(t)=f(j h) \psi_{j}(t)+f((j+1) h) \psi_{j+1}(t) \\
= & f(j h)\left(\frac{(j+1) h-t}{h}\right)+f(j h+h)\left(\frac{t-j h}{h}\right) \\
(\text { using }(6)) & \\
= & (j+1) f(j h)+t \frac{(f(j h+h)-f(j h))}{h} \\
& -j f(j h+h) .
\end{aligned}
$$

Expanding $f(t)$ in Taylor series at $t=j h$, we obtain

$$
f(t)=\sum_{r=0}^{\infty} \frac{(t-j h)^{r}}{r !} f^{(r)}(j h) .
$$

Thus, from (50) and (51), we get

$$
\begin{aligned}
|f(t)-\tilde{f}(t)|=\mid j h & \frac{(f(j h+h)-f(j h))}{h} \\
& -t \frac{(f(j h+h)-f(j h))}{h} \\
& +\sum_{r=1}^{\infty} \frac{(t-j h)^{r}}{r !} f^{(r)}(j h) \mid
\end{aligned}
$$

as $h \rightarrow 0$, and we have

$$
\begin{aligned}
|f(t)-\widetilde{f}(t)| & =\left|j h f^{\prime}(j h)-t f^{\prime}(j h)+\sum_{r=1}^{\infty} \frac{(t-j h)^{r}}{r !} f^{(r)}(j h)\right| \\
& =\left|\sum_{r=2}^{\infty} \frac{(t-j h)^{r}}{r !} f^{(r)}(j h)\right| \\
& \leq \frac{(t-j h)^{2}}{2 !}+O\left|(t-j h)^{3}\right| \leq \frac{n^{-2}}{2}+O\left(n^{-3}\right),
\end{aligned}
$$

as $(t-j h)<n^{-1}$, thus proving the following theorem.

Theorem 5. The absolute error $|f(t)-\tilde{f}(t)|$ associated with the approximation (49) is of the order $\mathrm{O}\left(n^{-2}\right)$.

\section{Numerical Results and Stability Analysis}

In this section, we discuss the implementation of our proposed algorithm and investigate its accuracy and stability by applying it on test functions with known analytical Abel inverse.

For, it is always desirable to test the behaviour of a numerical inversion method using simulated data for which the exact results are known, and thus making a comparison between inverted results and theoretical data is possible. We have tested our algorithm on several well-known test profiles that are commonly encountered in experimental data and widely used by researchers $[7,15,20]$. The accuracy of the proposed algorithm is demonstrated by calculating the parameters of absolute error $\Delta \eta\left(r_{i}\right)$, average deviation $\sigma$ also known as root mean square error (RMS). They are calculated using the following equations:

$$
\begin{gathered}
\Delta \eta\left(r_{i}\right)=\left|\eta_{a}\left(r_{i}\right)-\eta_{c}\left(r_{i}\right)\right|, \\
\sigma_{n+1}=\left\{\frac{1}{(n+1)} \sum_{i=0}^{n}\left[\eta_{a}\left(r_{i}\right)-\eta_{c}\left(r_{i}\right)\right]^{2}\right\}^{1 / 2} \\
=\left\{\frac{1}{(n+1)} \sum_{i=0}^{n}\left[\Delta \eta\left(r_{i}\right)\right]^{2}\right\}^{1 / 2}=\|\Delta \eta\|_{2},
\end{gathered}
$$

where $\eta_{c}\left(r_{i}\right)$ is the emission coefficient calculated at point $r_{i}$ using (48) and $\eta_{a}\left(r_{i}\right)$ is the exact analytical emissivity at the corresponding point. Note that $\sigma$, henceforth, denoted by $\sigma_{n+1}$ (for computational convenience) is the discrete $l^{2}$ norm of the absolute error $\Delta \eta$ denoted by $\|\Delta \eta\|_{2}$. Note that the calculation of $\sigma_{n+1}$ in (55) is performed by taking different values of $n$. In all the test profiles, the exact and noisy intensity profiles are denoted by $I_{1}(y)$ and $I_{1}^{\delta}(y)$, respectively, where $I_{1}^{\delta}(y)$ is obtained by adding a noise $\delta$ to $I_{1}(y)$ such that $I_{1}^{\delta}\left(y_{i}\right)=I_{1}\left(y_{i}\right)+\delta \theta_{i}$, where $y_{i}=i h, i=0,1,2, \ldots, n, n h=1$, and $\theta_{i}$ is the uniform random variable with values in $[-1,1]$ such that $\operatorname{Max}_{0 \leq i \leq n}\left|I_{1}^{\delta}\left(y_{i}\right)-I_{1}\left(y_{i}\right)\right| \leq \delta$.

The following test problems are solved with and without noise to illustrate the efficiency and stability of our method by choosing three different values of the noises $\delta_{k}$ as $\delta_{0}=0$, $\delta_{1}=\sigma_{n+1}$, and $\delta_{2}=p \%$ of $\mu_{n+1}$, where we mean $\mu_{n+1}=$ $(1 /(n+1)) \sum_{i=0}^{n} I_{1}(y)$. In each of the test problems given in this section we have taken positive parameter $\tau=0.0001$ and $p=0.1$, except for Example 10, where $\tau=0$ has been used. The absolute errors between exact and approximate emissivities, corresponding to different noises $\delta_{k}, k=0,1,2$ have been denoted by $E_{0}(r), E_{1}(r)$, and $E_{2}(r)$, respectively. In the text boxes of the figures, the notations $E 0(r), E 1(r)$, and $E 2(r)$ have been used for $E_{0}(r), E_{1}(r)$, and $E_{2}(r)$, respectively.

Though the stability of the algorithm is illustrated by various numerical experiments performed in this section, we analyze it also mathematically as follows.

The reconstructed emissivities $\eta_{c_{\delta}}(r)$ (with $\delta$ noise) and $\eta_{c_{0}}(r)$ (without noise) are obtained with and without noise term in the intensity profile $I_{1}(y)$, and using (48) these are given by

$$
\begin{aligned}
& \eta_{c_{\delta}}(r)=F_{n+1}^{\delta T}\left[a(y) P_{n+1}^{U}+b(y) P_{n+1}^{L}\right]^{-1} \bar{\Psi}_{n+1}(r), \\
& \eta_{c_{0}}(r)=F_{n+1}^{T}\left[a(y) P_{n+1}^{U}+b(y) P_{n+1}^{L}\right]^{-1} \bar{\Psi}_{n+1}(r),
\end{aligned}
$$


TABLE 1: Noise reduction $h(r)$ for $n=100$ at different values of $\delta, \tau=0, \gamma=1 / 2$.

\begin{tabular}{lccc}
\hline$r$ & $h(r)$ for $\delta=0.01$ & $h(r)$ for $\delta=0.001$ & $h(r)$ for $\delta=0.0001$ \\
\hline 0 & 0.019718 & 0.0019718 & 0.00019718 \\
0.1 & 0.0040696 & 0.00040696 & 0.000040696 \\
0.2 & 0.0035401 & 0.00035401 & 0.000035401 \\
0.3 & 0.0033118 & 0.00033118 & 0.000033118 \\
0.4 & 0.0032047 & 0.00032047 & 0.000032047 \\
0.5 & 0.0031726 & 0.00031726 & 0.000031726 \\
0.6 & 0.0032047 & 0.00032047 & 0.000032047 \\
0.7 & 0.0033118 & 0.00033118 & 0.000033118 \\
0.8 & 0.0035401 & 0.00035401 & 0.000035401 \\
0.9 & 0.0040696 & 0.00040696 & 0.000040696 \\
1 & 0.019718 & 0.0019718 & 0.00019718 \\
\hline
\end{tabular}

where $F_{n+1}^{\delta}$ and $F_{n+1}$ are known matrices, and they are obtained from the following equations:

$$
\begin{gathered}
I_{1}^{\delta}(y)=I_{1}(y)+\delta \theta_{i} \simeq F_{n+1}^{\delta T} \bar{\Psi}_{n+1}(y), \\
I_{1}(y) \simeq F_{n+1}^{T} \bar{\Psi}_{n+1}(y) .
\end{gathered}
$$

Hence

$$
\begin{aligned}
\eta_{c_{\delta}} & (r)-\eta_{c_{0}}(r) \\
\quad & =\left(F_{n+1}^{\delta T}-F_{n+1}^{T}\right)\left[a(y) P_{n+1}^{U}+b(y) P_{n+1}^{L}\right]^{-1} \bar{\Psi}_{n+1}(r) .
\end{aligned}
$$

Writing $H_{n+1}^{T}=F_{n+1}^{\delta T}-F_{n+1}^{T}$ and replacing random noise $\delta \theta_{i}$ by its maximum value $\delta$, we get

$$
\begin{aligned}
& \left\|\eta_{c_{\delta}}(r)-\eta_{c_{0}}(r)\right\| \\
& \quad=\left\|H_{n+1}^{T}\right\|\left\|\left[a(y) P_{n+1}^{U}+b(y) P_{n+1}^{L}\right]^{-1}\right\|\left\|\bar{\Psi}_{n+1}(r)\right\| .
\end{aligned}
$$

Let $h(r)=\eta_{c_{\delta}}(r)-\eta_{c_{0}}(r)=\left(F_{n+1}^{\delta T}-F_{n+1}^{T}\right)\left[a(y) P_{n+1}^{U}+\right.$ $\left.b(y) P_{n+1}^{L}\right]^{-1} \bar{\Psi}_{n+1}(r)$, then $h(r)$ reflects the noise reduction capability of the algorithm and its values at various points, and its graph is shown in Table 1 and Figure 1, respectively.

Table 1 demonstrates the noise filtering capability of the algorithm for three different noise outputs. From Table 1 and Figure 1 we see that noise reduction is symmetric about the point $r=0.5$, and the maximum reduction in noise is achieved at $r=0.5$ for all the three levels of noises $\delta=0.01$, 0.001 , and 0.0001 introduced in $I_{1}(y)$. The general behaviour of the noise reduction is the same irrespective of the value of $\delta$. In the interval $[0.02,0.98]$ the algorithm is stable, whereas the noise filtering capability decreases continuously and then jumps symmetrically in $[0,0.02) \cup(0.98,1]$.

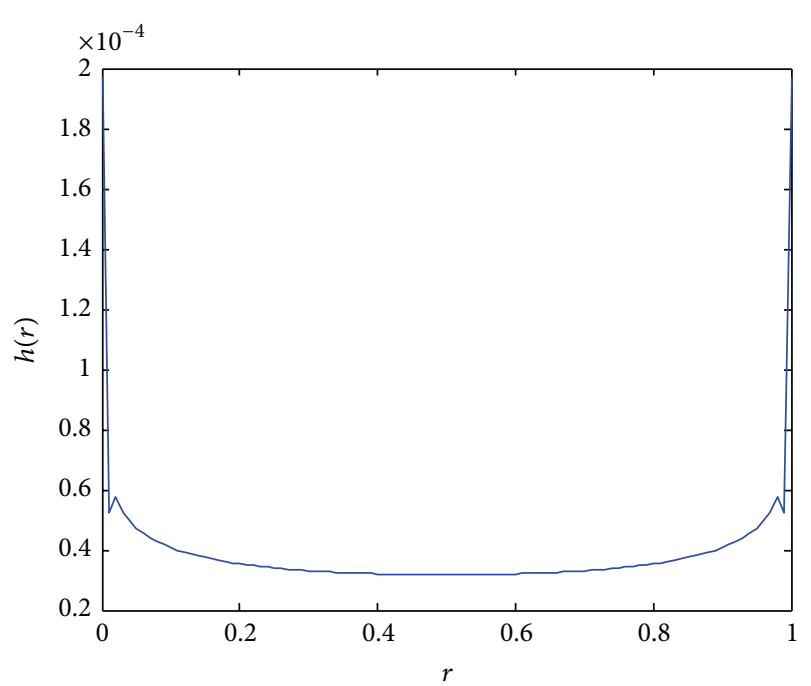

FIGURE 1: Noise reduction $h(r)$ for $n=100, \delta=0.0001, \tau=0$, and $\gamma=1 / 2$.

Example 6. Consider the generalized Abel integral equation:

$$
\begin{array}{r}
\frac{4}{3} y^{3 / 2}(1+y)+\frac{2}{3} y^{3 / 2}\left[2 \sqrt{(1-y) y^{3}}+\sqrt{y-y^{2}}\right] \\
=a(y) \int_{0}^{y} \frac{\eta(r)}{\sqrt{(y-r)}} d r+b(y) \int_{y}^{1} \frac{\eta(r)}{\sqrt{(r-y)}} d r, \\
0 \leq y \leq 1,
\end{array}
$$

where $a(y)=y+1, b(y)=y^{2}$, with the exact analytical solution $\eta(r)=r$.

The absolute errors $E_{k}(r)$ have been calculated for $n=$ 1000 and are given in Table 2 . The value of $\delta_{1}$ is $2.3829 \times 10^{-15}$, for $n=1000$. As $\delta_{2}=5.3347 \times 10^{-4}>10^{10} \delta_{1}$, the absolute error $E_{2}(r)$ is appreciably higher than $E_{0}(r)$ and $E_{1}(r)$. The Figure 2 compares the absolute errors $E_{0}(r)$ and $E_{1}(r)$ for noise $\delta_{1}=5.9827 \times 10^{-16}, n=100$.

Example 7. In this example, we consider the following Abel's integral equation $[7,20]$ :

$$
\begin{gathered}
\int_{0}^{y} \frac{\eta(r)}{\sqrt{(y-r)}} d r=y^{11 / 2}, \quad 0 \leq y \leq 1, \\
\text { with solution } \eta(r)=\frac{2^{10} 11[\Gamma(11 / 2)]^{2}}{2 \pi \Gamma(11)} r^{5} .
\end{gathered}
$$

The absolute errors corresponding to different noises are given in Table 3 . The values of various parameters are given as:

$\sigma_{3001}=9.4002 \times 10^{-8}\left(=\delta_{1}, n=3000\right), \sigma_{2001}=2.1116 \times$ $10^{-7}\left(=\delta_{1}, n=2000\right), \sigma_{1001}=8.4162 \times 10^{-7}\left(=\delta_{1}, n=\right.$ $1000), \mu_{3001}=0.1540, \mu_{2001}=0.1540$, and $\mu_{1001}=0.1542$. Taking $p=0.1$, the various values of respective $\delta_{2}$ are given in order as $1.5396 \times 10^{-4}, 1.5402 \times 10^{-4}$, and $1.5419 \times 10^{-4}$. 
TABLE 2: The absolute errors $E_{k}(r)$, at different nodal points $r$, for $n=1000$, in Example 6 .

\begin{tabular}{lcccccc}
\hline$r$ & 0.0 & 0.2 & 0.4 & 0.6 & 0.8 & 1.0 \\
\hline$E_{0}(r)$ & 0 & $7.494 \times 10^{-16}$ & $1.2212 \times 10^{-15}$ & $1.8874 \times 10^{-15}$ & $8.1046 \times 10^{-15}$ & $1.5543 \times 10^{-15}$ \\
$E_{1}(r)$ & $1.132 \times 10^{-13}$ & $2.4591 \times 10^{-14}$ & $8.3822 \times 10^{-15}$ & $1.7764 \times 10^{-14}$ & $2.931 \times 10^{-14}$ & $3.5194 \times 10^{-14}$ \\
$E_{2}(r)$ & 0.011106 & 0.0064263 & 0.0025148 & 0.01039 & 0.00029049 & 0.00014606 \\
\hline
\end{tabular}

TABLE 3: The absolute errors $E_{k}(r)$, at different nodal points $r$, for $n=3000,2000$, and 1000 .

\begin{tabular}{cccccccc}
\hline & $n$ & $r=0.0$ & $r=0.2$ & $r=0.4$ & $r=0.6$ & $r=0.8$ & $r=1.0$ \\
\hline \multirow{4}{*}{$E_{0}(r)$} & 3000 & 0 & $1.9712 \times 10^{-9}$ & $1.5849 \times 10^{-8}$ & $5.361 \times 10^{-8}$ & $1.2724 \times 10^{-7}$ & $2.4875 \times 10^{-7}$ \\
& 2000 & 0 & $4.4178 \times 10^{-7}$ & $3.5563 \times 10^{-8}$ & $1.2035 \times 10^{-7}$ & $2.8575 \times 10^{-7}$ & $5.5872 \times 10^{-7}$ \\
& 1000 & 0 & $1.7515 \times 10^{-8}$ & $1.4137 \times 10^{-7}$ & $4.7899 \times 10^{-7}$ & $1.138 \times 10^{-6}$ & $2.2262 \times 10^{-6}$ \\
\hline \multirow{4}{*}{$E_{1}(r)$} & 3000 & $4.6073 \times 10^{-6}$ & $1.5029 \times 10^{-6}$ & $2.5454 \times 10^{-6}$ & $5.3295 \times 10^{-7}$ & $7.3491 \times 10^{-7}$ & $2.0451 \times 10^{-6}$ \\
& 2000 & $1.0031 \times 10^{-5}$ & $1.1159 \times 10^{-6}$ & $4.5957 \times 10^{-6}$ & $2.3615 \times 10^{-7}$ & $4.4563 \times 10^{-6}$ & $9.2247 \times 10^{-7}$ \\
& 1000 & $1.7061 \times 10^{-5}$ & $7.7631 \times 10^{-6}$ & $7.528 \times 10^{-6}$ & $4.4903 \times 10^{-6}$ & $1.5261 \times 10^{-5}$ & $4.2806 \times 10^{-6}$ \\
\hline \multirow{3}{*}{$E_{2}(r)$} & 3000 & 0.0061012 & 0.0011742 & 0.0011595 & 0.0039968 & 0.00064593 & 0.0029774 \\
& 2000 & 0.0068177 & 0.0068177 & 0.00078732 & 0.00067568 & 0.0016351 & $4.2642 \times 10^{-5}$ \\
& 1000 & 0.0001341 & 0.00067286 & 0.0010734 & 0.00040813 & 0.00098541 & 0.00074723 \\
\hline
\end{tabular}

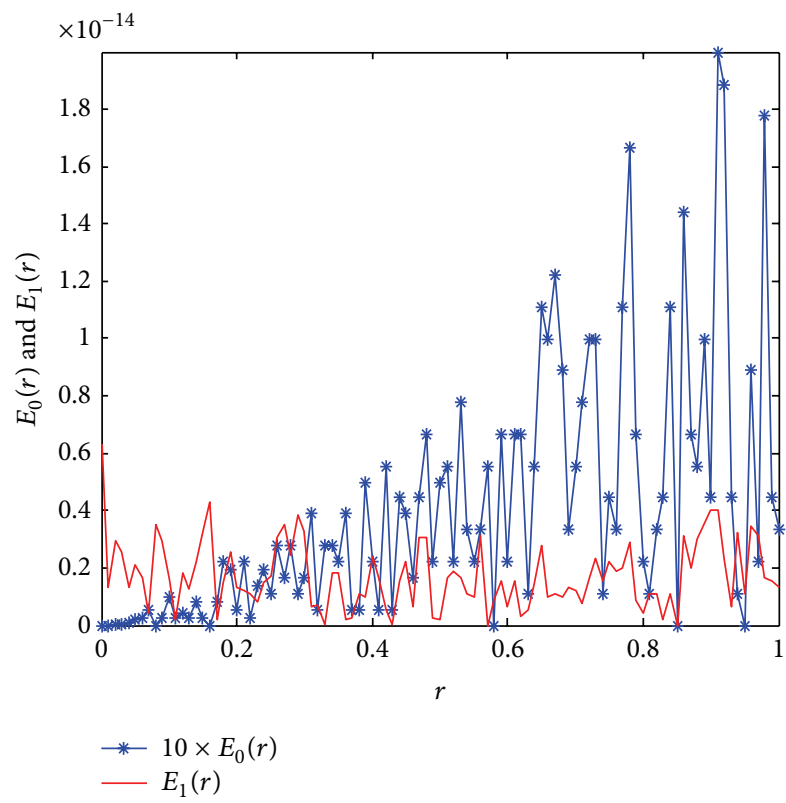

FIGURE 2: Absolute errors $E_{0}(r)$ and $E_{1}(r)$ for $n=100, \delta_{1}=5.9827 \times$ $10^{-16}$.

In Figure 3, the exact and reconstructed emissivities (with $\delta_{2}$ noise) have been shown for $n=50$, and the two emissivities match very well even for higher noise $\delta_{2}$ introduced in the intensity profile. For $n=100$, Figures 4 and 5 show the absolute errors $E_{0}(r), E_{1}(r)$ and $E_{0}(r), E_{2}(r)$, respectively.

Example 8. In this example we consider the following Abel's integral equation [22]:

$$
\int_{0}^{y} \frac{\eta(r)}{\sqrt{(y-r)}} d r=I_{1}(y), \quad 0 \leq y \leq 1,
$$

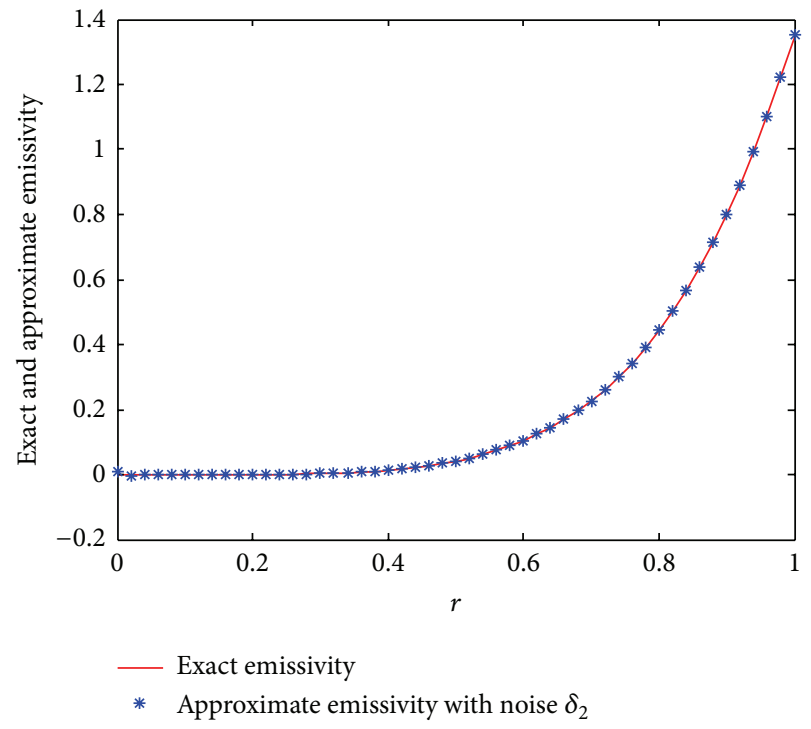

FIGURE 3: Exact and approximate emissivities with noise $\delta_{2}=$ $1.6081 \times 10^{-4}$ for $n=50$.

where

$$
I_{1}(y)= \begin{cases}\frac{4}{3} y^{3 / 2}, & 0 \leq y<\frac{1}{2} \\ \frac{4}{3} y^{3 / 2}-\frac{8}{3}\left(y-\frac{1}{2}\right)^{3 / 2}, & \frac{1}{2} \leq y \leq 1\end{cases}
$$

The exact solution of the integral equation (62) is given by

$$
\eta(r)= \begin{cases}r, & 0 \leq r<\frac{1}{2}, \\ 1-r, & \frac{1}{2} \leq r \leq 1 .\end{cases}
$$

In Table 4, the absolute errors for different noises have been shown. Various parameters used for Table 4 are 
TABLE 4: The absolute errors $E_{k}(r)$, at different nodal points $r$, for $n=1000$, in Example 8.

\begin{tabular}{lcccccc}
\hline$r$ & 0.0 & 0.2 & 0.4 & 0.6 & 0.8 & 1.0 \\
\hline$E_{0}(r)$ & 0 & $4.4409 \times 10^{-16}$ & $5.5511 \times 10^{-17}$ & $2.7200 \times 10^{-15}$ & $2.1649 \times 10^{-15}$ & $3.3888 \times 10^{-15}$ \\
$E_{1}(r)$ & $5.5359 \times 10^{-14}$ & $7.8271 \times 10^{-15}$ & $2.2093 \times 10^{-14}$ & $1.1768 \times 10^{-14}$ & $1.4433 \times 10^{-14}$ & $1.5344 \times 10^{-14}$ \\
$E_{2}(r)$ & 0.0042764 & 0.0018814 & $3.4439 \times 10^{-5}$ & 0.0014343 & 0.0024313 & 0.00018694 \\
\hline
\end{tabular}

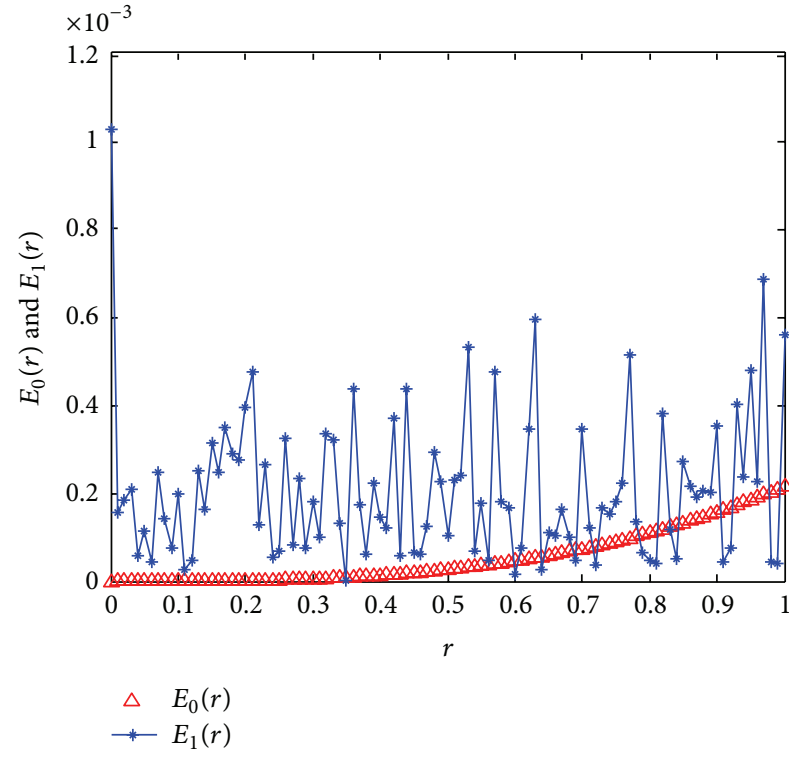

FIGURE 4: Absolute errors $E_{0}(r)$ and $E_{1}(r)$ for $\delta_{1}=8.2435 \times 10^{-5}$, $n=100$.

$\sigma_{1001}=1.8576 \times 10^{-15}=\delta_{1}, \mu_{1001}=0.3446$, and $\delta_{2}=3.4462 \times$ $10^{-4}$. Figure 6 shows the graph of exact and approximate emissivities $\eta(r)$ (without noise) for $n=50$. Absolute errors $E_{0}(r)$ and $E_{1}(r)$, for $n=50$ and $n=100$, are shown in Figures 7 and 8 , respectively.

Example 9. Consider the generalized Abel integral equation:

$$
\begin{aligned}
I_{1}(y)= & e^{y} \sin (y) \int_{0}^{y} \frac{\eta(r)}{(y-r)^{1 / 3}} d r \\
& +e^{-y} \cos (y) \int_{y}^{1} \frac{\eta(r)}{(r-y)^{1 / 3}} d r, \quad 0<y<1,
\end{aligned}
$$

where $I_{1}(y)=e^{y} \sin (y) y^{5 / 3}+e^{-y} \cos (y)(1-y)^{2 / 3}(2+3 y)$. The exact solution of $(65)$ is $\eta(r)=10 r / 9$. In Figure 9, the comparison between $E_{0}(r)$ and $E_{1}(r)$ is shown, for $n=100$.

Example 10. For the pair $[14,15,23]$ :

$$
\begin{gathered}
\eta(r)=(1-r)^{2}(1+12 r), \quad \text { for } 0 \leq r \leq 1, \\
I_{1}(y)=\left[\left(\frac{384}{35} y^{7 / 2}-\frac{368}{15} y^{5 / 2}+\frac{40}{3} y^{3 / 2}+2 \sqrt{y}\right)\right. \\
\left.+\frac{16}{105}(1-y)^{5 / 2}(19+72 y)\right]
\end{gathered}
$$

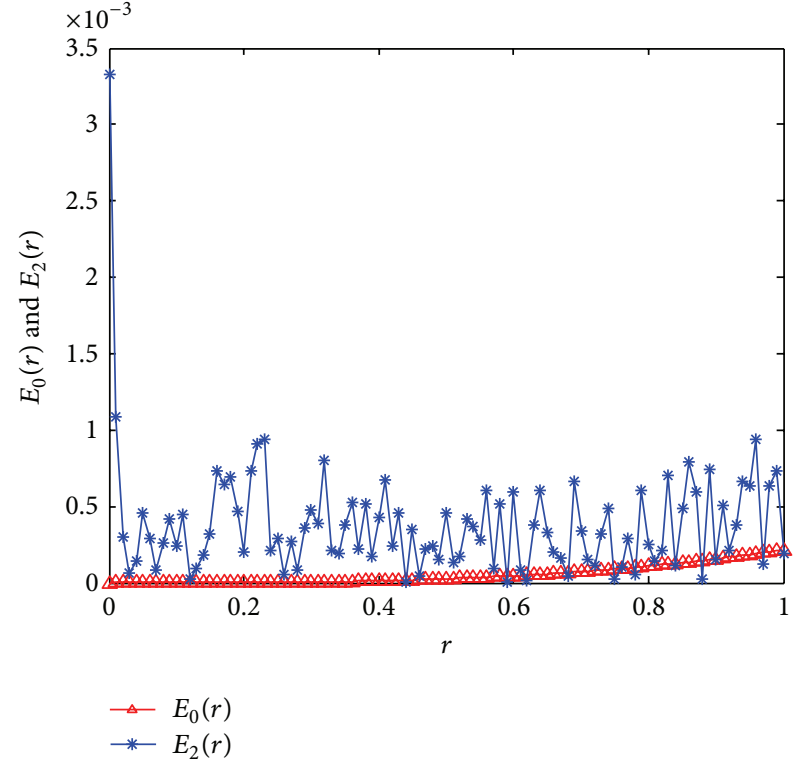

Figure 5: Absolute errors $E_{0}(r)$ and $E_{2}(r)$ for $\delta_{2}=1.5732 \times 10^{-4}$, $n=100$.

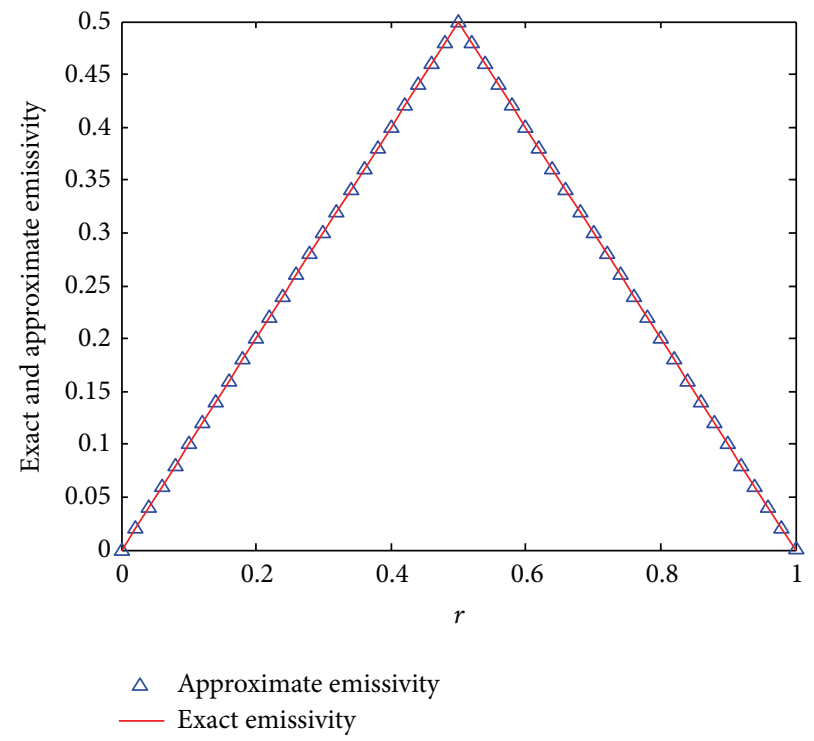

FIGURE 6: Exact and approximate solutions for $n=50$.

with $a(y)=b(y)=1$, and the various parameters are as follows:

$\delta_{1}=2.1111 \times 10^{-7}(n=3000), \delta_{1}=4.7384 \times$ $10^{-7}(n=2000), \delta_{1}=1.8852 \times 10^{-6}(n=1000)$, and $\delta_{2}=0.0036$, for all the three chosen values of $n$. 
TABLE 5: The absolute errors $E_{k}(r)$, at different nodal points $r$, for $n=3000,2000$, and 1000 .

\begin{tabular}{cccccccc}
\hline & $n$ & $r=0.0$ & $r=0.2$ & $r=0.4$ & $r=0.6$ & $r=0.8$ & $r=1.0$ \\
\hline \multirow{3}{*}{$E_{0}(r)$} & 3000 & $5.4281 \times 10^{-7}$ & $2.8998 \times 10^{-7}$ & $1.5818 \times 10^{-7}$ & $2.6121 \times 10^{-8}$ & $1.0578 \times 10^{-7}$ & $2.9684 \times 10^{-7}$ \\
& 2000 & $1.2088 \times 10^{-6}$ & $6.5113 \times 10^{-7}$ & $3.5535 \times 10^{-7}$ & $5.887 \times 10^{-8}$ & $2.3717 \times 10^{-7}$ & $6.5839 \times 10^{-7}$ \\
& 1000 & $4.7364 \times 10^{-6}$ & $2.5925 \times 10^{-6}$ & $1.4164 e \times 10^{-6}$ & $2.3637 \times 10^{-7}$ & $9.4123 \times 10^{-7}$ & $2.559 \times 10^{-6}$ \\
\hline \multirow{3}{*}{$E_{1}(r)$} & 3000 & $6.8261 \times 10^{-6}$ & $2.1337 \times 10^{-6}$ & $2.2043 \times 10^{-6}$ & $1.4765 \times 10^{-6}$ & $1.4642 \times 10^{-6}$ & $3.421 \times 10^{-6}$ \\
& 2000 & $1.589 \times 10^{-6}$ & $5.7191 \times 10^{-6}$ & $2.8577 \times 10^{-6}$ & $2.5629 \times 10^{-6}$ & $4.4183 \times 10^{-6}$ & $3.9074 \times 10^{-8}$ \\
& 1000 & $1.9156 \times 10^{-5}$ & $4.4716 \times 10^{-6}$ & $1.643 \times 10^{-5}$ & $1.712 \times 10^{-5}$ & $3.6022 \times 10^{-6}$ & $3.6477 \times 10^{-6}$ \\
\hline \multirow{3}{*}{$E_{2}(r)$} & 3000 & 0.01495 & 0.020628 & 0.021094 & 0.065933 & 0.011844 & 0.095356 \\
& 2000 & 0.022427 & 0.010769 & 0.024946 & 0.014392 & 0.0068535 & 0.028831 \\
& 1000 & 0.056845 & 0.033342 & 0.013319 & 0.018905 & 0.028086 & 0.045314 \\
\hline
\end{tabular}

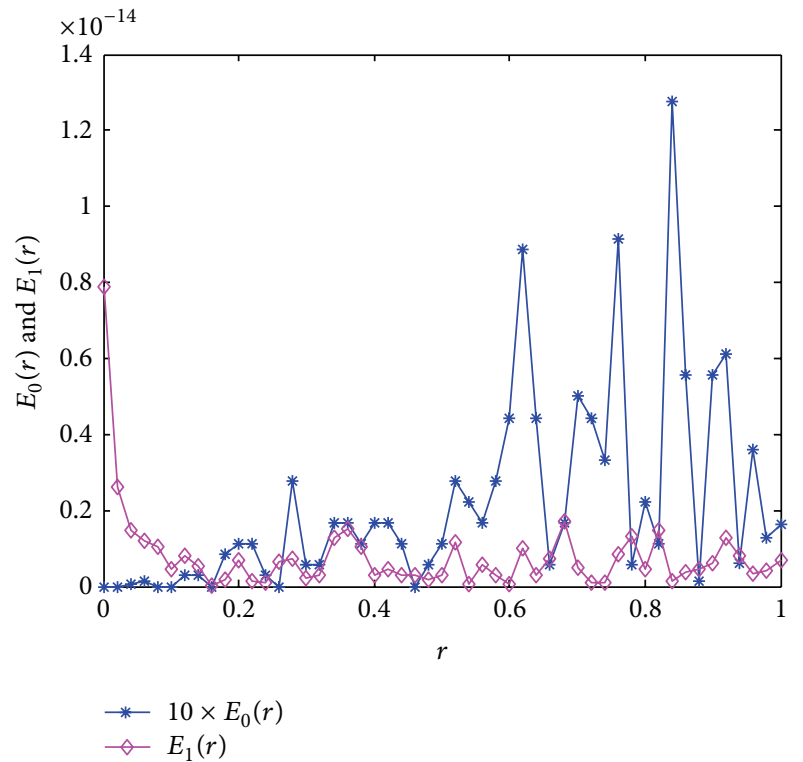

FIGURE 7: Absolute errors $E_{0}(r)$ and $E_{1}(r)$ for $\delta_{1}=3.4218 \times 10^{-16}$, $n=50$.

The absolute errors corresponding to different noises are given in Table 5. Figure 10 shows the exact and approximate emissivities (without noise and with noise $\delta_{2}=0.0036$ ) whereas, in Figure 11, a comparison between $E_{0}(r)$ and $E_{1}(r)$ is shown for $\delta_{1}=1.8232 \times 10^{-4}, n=100$.

Example 11. Consider the generalized Abel integral equation (15) with $\gamma=1 / 2, a(y)=(3 / 4) \exp (y), b(y)=\exp (2 y)+$ $(1 / \sqrt{2 \pi})$, for the pair $\eta(r)=\sin r$ and

$$
\begin{aligned}
& I_{1}(y) \\
& =\left[\begin{array}{c}
\exp (y) y_{1}^{3 / 2} F_{2}\left(1, \frac{5}{4}, \frac{7}{4},-\frac{y^{2}}{4}\right)+\sqrt{2 \pi}\left(\exp (2 y)+\frac{1}{\sqrt{2 \pi}}\right) \\
\left(C\left(\frac{\sqrt{2-2 y}}{\sqrt{\pi}}\right) \sin y+S\left(\frac{\sqrt{2-2 y}}{\sqrt{\pi}}\right) \cos y\right)
\end{array}\right],
\end{aligned}
$$

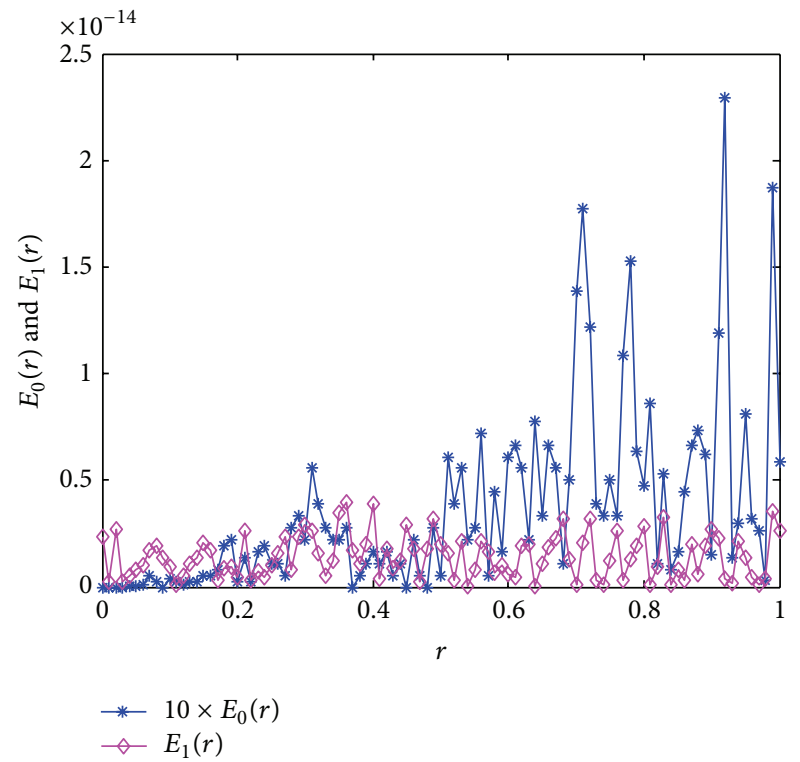

FIGURE 8: Absolute errors $E_{0}(r), E_{1}(r)$ for $\delta_{1}=5.5794 \times 10^{-16}, n=$ 100.

where $C(z)$ and $S(z)$ in (67) are called Fresnel integrals. These are defined as

$$
C(z)=\int_{0}^{z} \cos \left(\frac{\pi t^{2}}{2}\right) d t, \quad S(z)=\int_{0}^{z} \sin \left(\frac{\pi t^{2}}{2}\right) d t .
$$

For, $n=3000$, different absolute errors are given in Table 6 . The various parameters for $n=3000$ are $\delta_{1}=0.0173$ and $\delta_{2}=7.2830 \times 10^{-4}$. In Figure 12, the exact and approximate emissivities (without noise) are shown for $n=100$.

\section{Conclusions}

We have constructed operational matrices of integration based on extended hat functions and used them to propose a stable algorithm for the numerical inversion of the generalized Abel integral equation. The earlier numerical 
TABLE 6: The absolute errors $E_{k}(r)$ for $n=3000$, for Example 11.

\begin{tabular}{lcccccc}
\hline$r$ & 0.0 & 0.2 & 0.4 & 0.6 & 0.8 & 0.9 \\
\hline$E_{0}(r)$ & $3.389 \times 10^{-7}$ & $4.7548 \times 10^{-7}$ & $7.3287 \times 10^{-7}$ & $1.3451 \times 10^{-6}$ & $3.7972 \times 10^{-6}$ & $1.0734 \times 10^{-5}$ \\
$E_{1}(r)$ & 0.38495 & 0.079121 & 0.17527 & 0.096373 & 0.40061 & 0.050512 \\
$E_{2}(r)$ & 0.0066376 & 0.0058214 & 0.0070449 & 0.018136 & 0.013987 & 0.0021626 \\
\hline
\end{tabular}

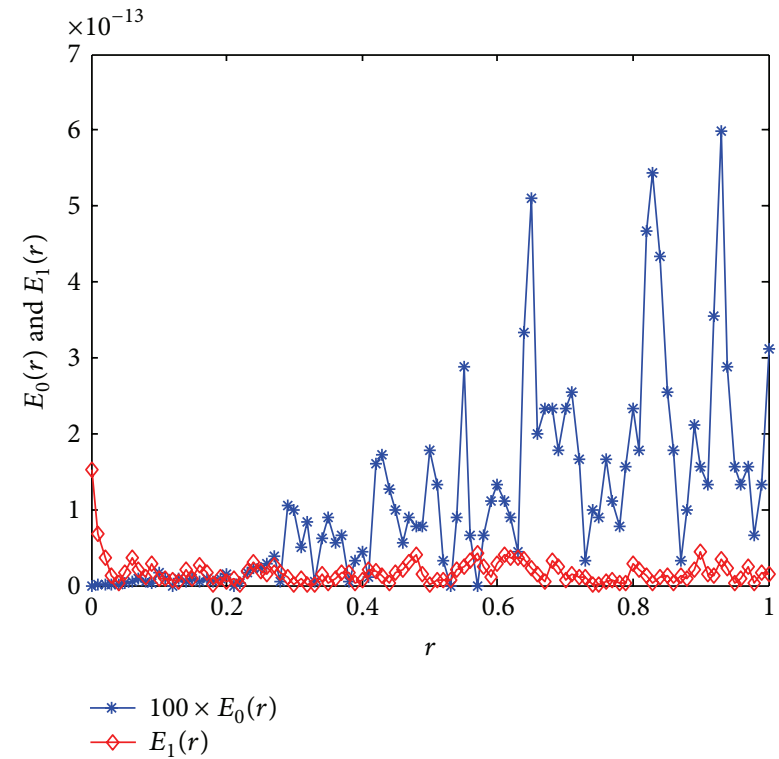

FIGURE 9: Comparison between $E_{0}(r)$ and $E_{1}(r)$ for $\delta_{1}=1.6911 \times$ $10^{-15}, n=100$.

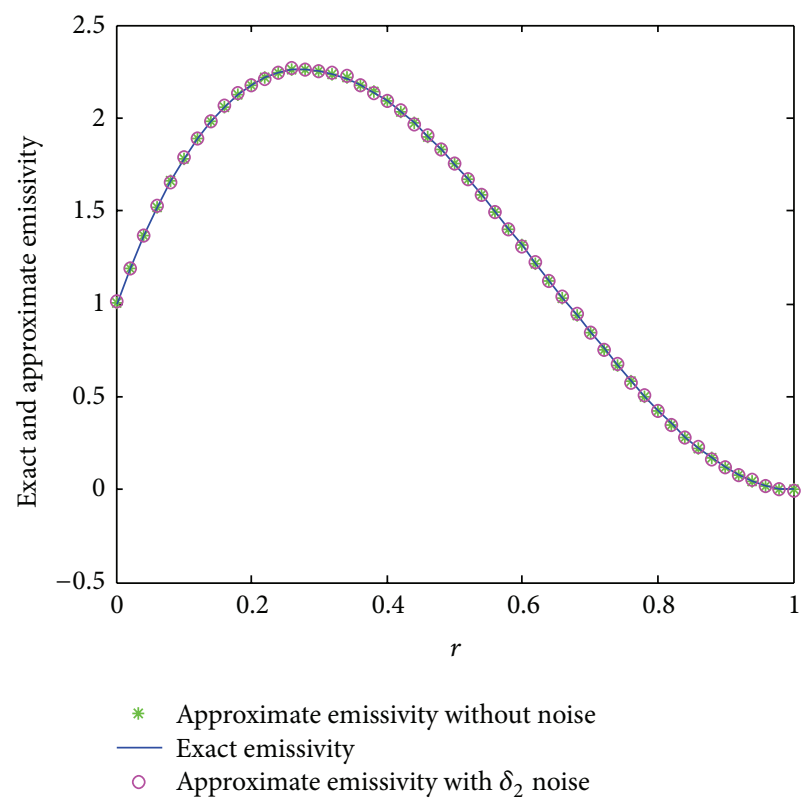

FIGURE 10: Exact and approximate emissivities (without noise and with noise $\delta_{2}=0.0036$ ) for $n=50$.

inversions were restricted to a part of the general Abel's integral equation. We have extended the hat function beyond their domain $[0,1]$ to avoid the singularity of the matrix at $t=0,1$. These operational matrices are given by the general formulae (39) and (44), thus making the evaluation of

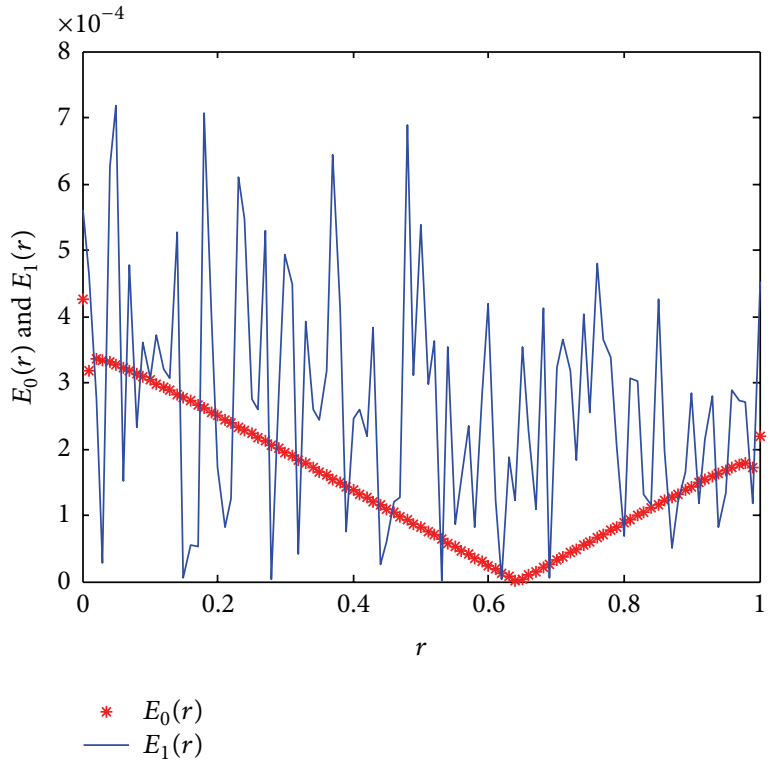

FIgURE 11: Comparison between $E_{0}(r)$ and $E_{1}(r)$ for $\delta_{1}=1.8232 \times$ $10^{-4}, n=100$.

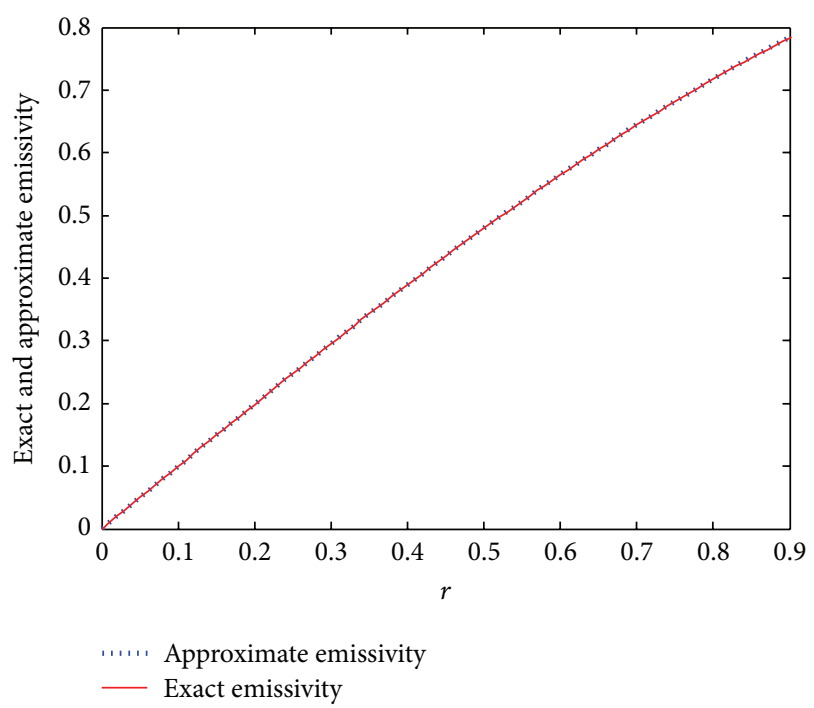

FIGURE 12: Exact and approximate emissivities (without noise) for $n=100$.

these matrices of any order extremely easy whereas in case of Bernstein operational matrices no such formula was available $[19,20]$. The stability with respect to the data is restored and good accuracy is obtained even for high noise levels in the data. An error analysis and stability analysis are also given. 


\section{Acknowledgments}

The authors are grateful to the learned reviewer for his valuable suggestions which have led to the improvement of the paper in the present form. Also, the first author acknowledges the financial support from UGC New-Delhi, India, under Faculty Improvement Program (FIP), whereas the second and the third authors acknowledge the financial support from UGC and CSIR New-Delhi, India, respectively, under JRF schemes.

\section{References}

[1] N. H. Abel, "Resolution d'un problem de mechanique," Journal Für Die Reine Und Angewandte Mathematik, vol. 1, pp. 153-157, 1826.

[2] L. Mach, Wien. Akad. Ber. Math. Phys. Klasse, vol. 105, p. 605, 1896.

[3] H. R. Griem, Plasma Spectroscopy, McGraw-Hill, New York, NY, USA, 1963.

[4] F. G. Tricomi, Integral Equations, Wiley-Interscience, New York, NY, USA, 1975.

[5] R. S. Anderssen, "Stable procedures for the inversion of Abel's equation," Journal of the Institute of Mathematics and its Applications, vol. 17, no. 3, pp. 329-342, 1976.

[6] I. Beniaminy and M. Deutsch, "ABEL: stable, high accuracy program for the inversion of Abel's integral equation," Computer Physics Communications, vol. 27, no. 4, pp. 415-422, 1982.

[7] S. Bhattacharya and B. N. Mandal, "Use of Bernstein polynomials in numerical solutions of Volterra integral equations," Applied Mathematical Sciences, vol. 2, no. 33-36, pp. 1773-1787, 2008.

[8] C. J. Cremers and R. C. Birkebak, "Application of the Abel integral equation to spectroscopic data," Applied Optics, vol. 5, pp. 1057-1064, 1966.

[9] M. Deutsch and I. Beniaminy, "Derivative-free inversion of Abel's integral equation," Applied Physics Letters, vol. 41, no. 1, pp. 27-28, 1982.

[10] M. Deutsch, "Abel inversion with a simple analytic representation for experimental data," Applied Physics Letters, vol. 42, no. 3, pp. 237-239, 1983.

[11] R. Grenflo, "Computation of rough solutions of Abel integral equation," in Inverse ILL-Posed Problems, H. W. Engel and C. W. Groetsch, Eds., pp. 195-210, Academic Press, NewYork, NY, USA, 1987.

[12] L. M. Ignjatović and A. A. Mihajlov, "The realization of Abel's inversion in the case of discharge with undetermined radius," Journal of Quantitative Spectroscopy \& Radiative Transfer, vol. 72, no. 5, pp. 677-689, 2002.

[13] J.-P. Lanquart, "Error attenuation in Abel inversion," Journal of Computational Physics, vol. 47, no. 3, pp. 434-443, 1982.

[14] S. Ma, H. Gao, L. Wu, and G. Zhang, "Abel inversion using Legendre polynomials approximations," Journal of Quantitative Spectroscopy \& Radiative Transfer, vol. 109, no. 10, pp. 1745-1757, 2008.

[15] S. Ma, H. Gao, G. Zhang, and L. Wu, "Abel inversion using Legendre wavelets expansion," Journal of Quantitative Spectroscopy \& Radiative Transfer, vol. 107, no. 1, pp. 61-71, 2007.

[16] G. N. Minerbo and M. E. Levy, "Inversion of Abel's integral equation by means of orthogonal polynomials," SIAM Journal on Numerical Analysis, vol. 6, pp. 598-616, 1969.
[17] D. A. Murio, D. G. Hinestroza, and C. E. Mejía, "New stable numerical inversion of Abel's integral equation," Computers and Mathematics with Applications, vol. 23, no. 11, pp. 3-11, 1992.

[18] M. Sato, "Inversion of the Abel integral equation by use of simple interpolation formulas," Contributions to Plasma Physics, vol. 25, pp. 573-577, 1985.

[19] V. K. Singh, R. K. Pandey, and O. P. Singh, "New stable numerical solutions of singular integral equations of Abel type by using normalized Bernstein polynomials," Applied Mathematical Sciences, vol. 3, no. 5-8, pp. 241-255, 2009.

[20] O. P. Singh, V. K. Singh, and R. K. Pandey, "A stable numerical inversion of Abel's integral equation using almost Bernstein operational matrix," Journal of Quantitative Spectroscopy \& Radiative Transfer, vol. 111, pp. 245-252, 2010.

[21] A. Chakrabarti, "Solution of the generalized Abel integral equation," Journal of Integral Equations and Applications, vol. 20, no. 1, pp. 1-11, 2008.

[22] L. Huang, Y. Huang, and X.-F. Li, "Approximate solution of Abel integral equation," Computers and Mathematics with Applications, vol. 56, no. 7, pp. 1748-1757, 2008.

[23] M. J. Buie, J. T. P. Pender, J. P. Holloway, T. Vincent, P. L. G. Ventzek, and M. L. Brake, "Abel's inversion applied to experimental spectroscopic data with off axis peaks," Journal of Quantitative Spectroscopy \& Radiative Transfer, vol. 55, no. 2, pp. 231-243, 1996. 


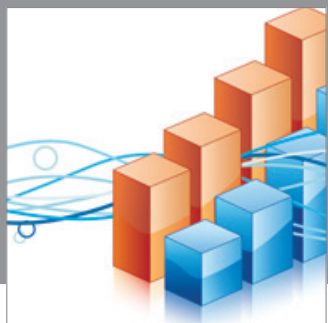

Advances in

Operations Research

mansans

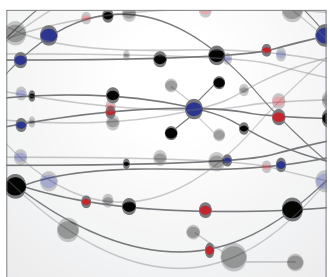

The Scientific World Journal
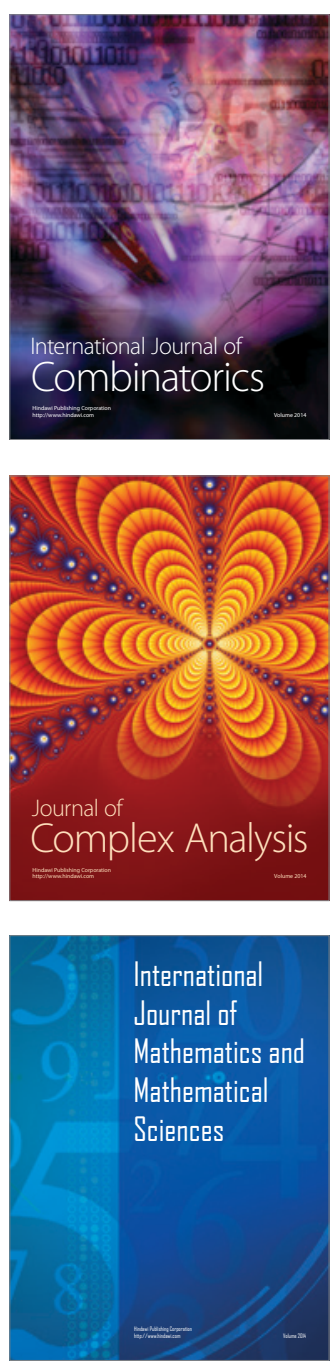
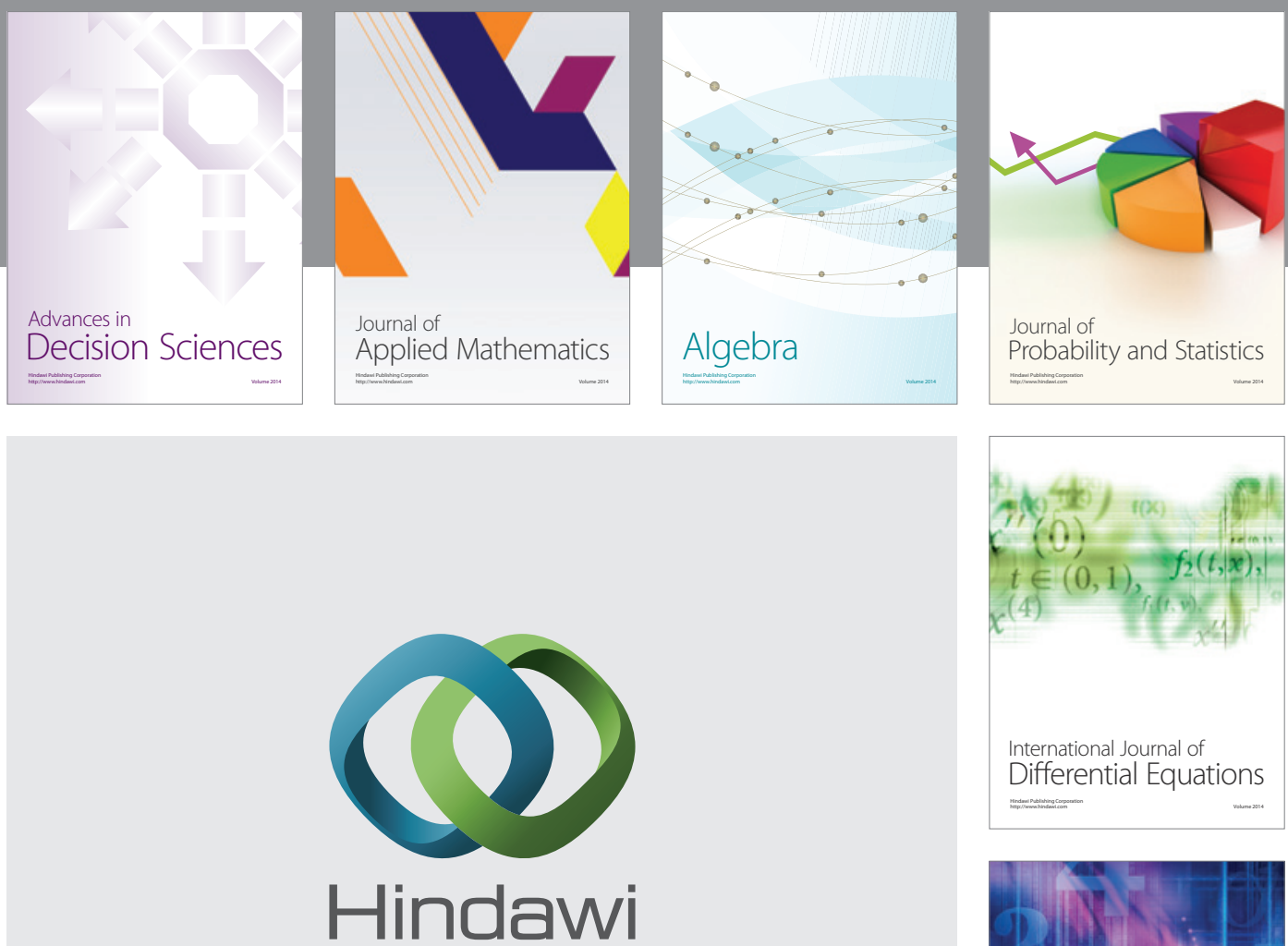

Submit your manuscripts at http://www.hindawi.com
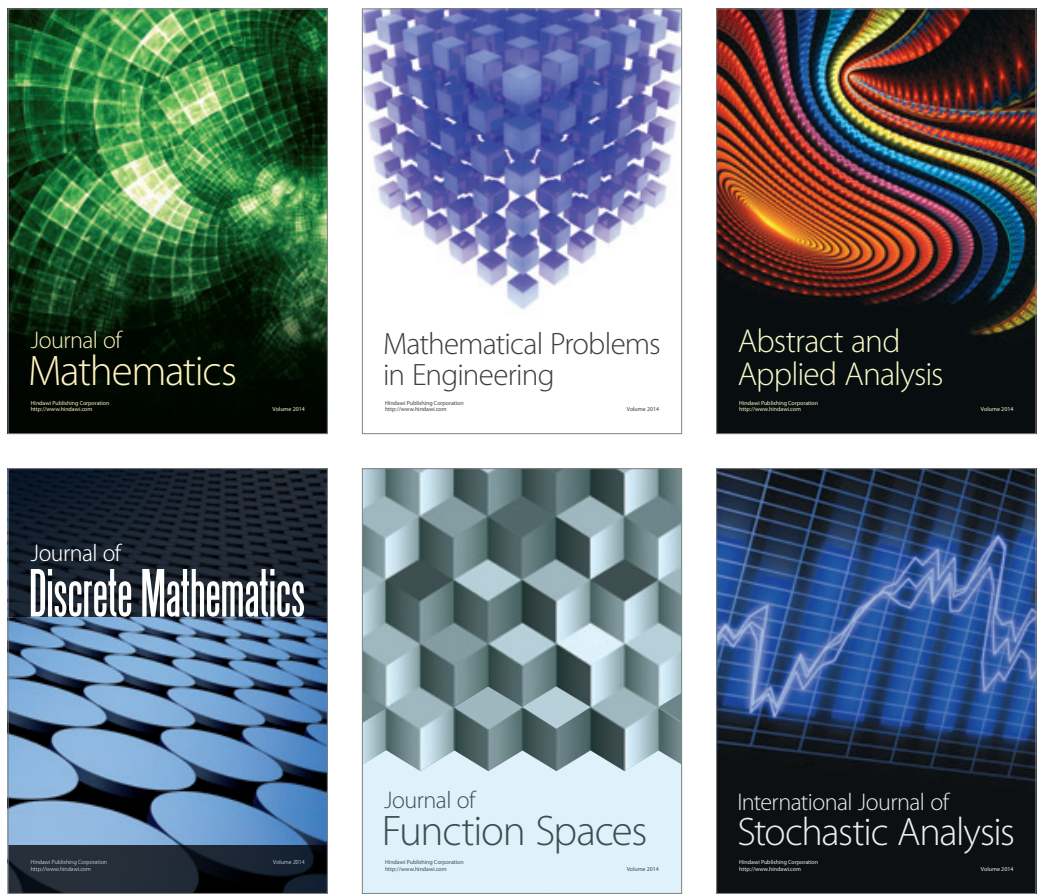

Journal of

Function Spaces

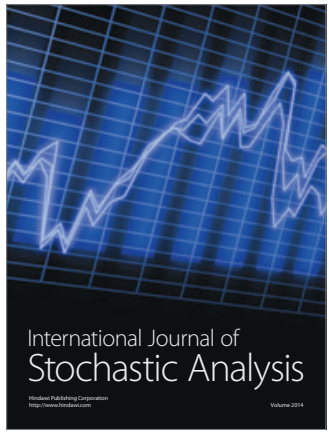

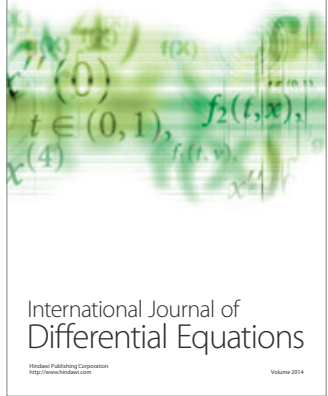
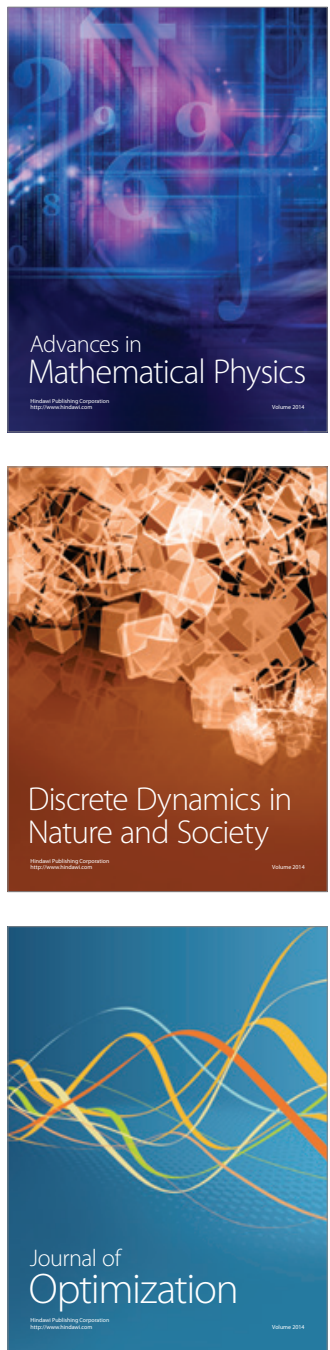